\title{
Use of machine learning techniques in the prediction of credit recovery
}

\author{
Rogerio Gomes Lopes ${ }^{*}, 1$,Marcelo Ladeira ${ }^{2}$, Rommel Novaes Carvalho ${ }^{2}$ \\ ${ }^{1}$ Bank of Brazil, IT Department,Brazil,rglopes@bb.com.br \\ ${ }^{2}$ University of Brasilia, Department of Computer Science,Brasilia,Brazil, mladeira@unb.br, rommel.carvalho@gmail.com
}

\begin{tabular}{l} 
A R T I C L E I N F O \\
\hline Article history: \\
Received: 04 June, 2017 \\
Accepted: 28 July, 2017 \\
Online: 10 August, 2017 \\
\hline Keywords: \\
machine learning \\
data mining \\
credit recovery \\
h2o.ai \\
\hline
\end{tabular}

\section{Introduction}

This paper is an extension of the work originally presented at the International Conference on Machine Learning and Application (ICMLA 2016) [1], which presented the first results of a Brazilian bank research to reduce its losses with defaulting clients. That study covered only a sample of 22.764 transactions, representing a homogeneous group of bank customers. We extend our previous work by adding all operations from individual costumers which were in arrears in July 2016.

The Figure 1 shows that there was a slight decrease in the number of debtors in June 2016, but increased again in the following months.

The Bank had nearly 54 million active credit agreements with individuals at the end of July 2016. Of this amount, approximately 8.6 million were delayed for 15 days or more, accounting for $15.9 \%$ of the contracts. These delinquent contracts amounted to more than R $\$ 20.8$ billion (US $\$ 6.4$ billion in July 2016), accounting for approximately $5.8 \%$ of the Bank's individuals loan portfolio, an increase of 1.2 percentage points over December 2014. That is, in 21 months the financial volume of overdue loans contracted by individuals increased by $26 \%$.

The Brazilian Central Bank (BACEN) regulation requires financial institutions to classify their credit operations and perform a Provision for Doubtful Accounts (PDA), according to a risk classification. The main criteria for the classification is the number of days in arrears of each individual credit agreement.
The Table 1 shows the days-in-delay ranges considered to determine a risk classification and therefore the minimum percentage PDA that financial institutions must reserve. As an operation increases the number of days in arrears, there is a non-linear increase of PDA, which may allocate $100 \%$ of the outstanding balance of the contract. For example, an operation with a debit balance of $R \$ 1,000$, with 15 days in arrears, must reserve a minimum provision of $R \$$ 10 . The amount of the provision may reach $R \$ 1,000$ if the arrear reach 180 days.

Table 1: Days in arrears $x$ Provision

\begin{tabular}{|l|c|r|}
\hline Days in arrears & Minimum Risk & PDA \% \\
\hline $15-30$ & $\mathrm{~B}$ & 1 \\
\hline $31-60$ & $\mathrm{C}$ & 3 \\
\hline $61-90$ & $\mathrm{D}$ & 10 \\
\hline $91-120$ & $\mathrm{E}$ & 30 \\
\hline $121-150$ & $\mathrm{~F}$ & 50 \\
\hline $151-180$ & $\mathrm{G}$ & 70 \\
\hline over 180 & $\mathrm{H}$ & 100 \\
\hline
\end{tabular}

At the time of the credit granting, financial institutions assume the credit risk and make the corresponding provisions in accordance with the current Central Bank regulation. Acting in this way, in a possible default of the customer, the financial institution and the stability of the financial system will be protected. However, as a customer delays its operations, the natural reaction of financial institutions is to restrict credit to them, increasing the chances of these

\footnotetext{
Rogerio Gomes Lopes, Braslia, Brazil, rglopes@bb.com.br.
} 


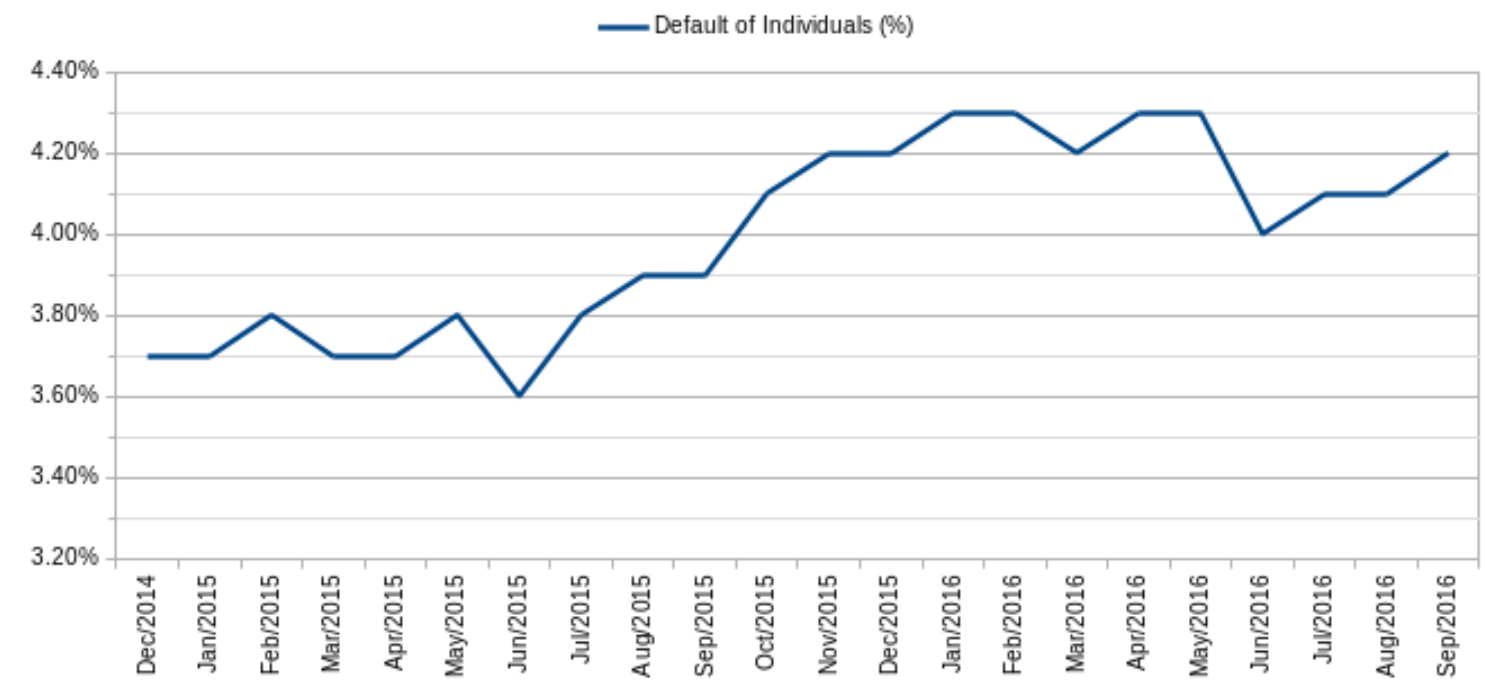

Figure 1: Default of individuals.

customer's evasion to other institutions, since they will not be able to carry out new credit operations with the original institution.

With the increase in delinquency, a mobilization of account managers of the bank began in order to mitigate the evasion of its clients by approaching the customer in arrear and proposing alternatives that could fix the delayed payments. Hence, solving the default situation and the possible loss of the customer of its portfolio, as well as reducing the financial amount allocated to (PDA).

Provided that the selection of the clients is a time and resource consuming task, the main objective of this study was to apply machine learning techniques to predict the recovery probability of credit transactions, providing a list of delinquent clients with the greatest potential for regularization of their operations.

Models were developed using Generalized Linear Models (GLM), Gradient Boosted Methods (GBM), Distributed Random Forest (DRF) and Deep Learning $(\mathrm{DL})^{1}$. The models were compared using the recall indicator, which will be explained on section 3 . The models were developed using the $\mathrm{R}$ language and $\mathrm{H} 2 \mathrm{O}$ machine learning platform, considering its parallel processing capabilities. Further details on section 3.2

This paper is organized as follows: Section 2 presents the credit scoring state of the art. Section 3 presents the methodology used in this study. Section 4 presents the modeling and evaluation of the generated models for each method. Section 5 presents the conclusion and future works.

\section{State of the Art}

The default numbers observed in Brazil, from December 2014 to September 2016, indicate that financial institutions need a tool to support their credit granting decisions. Although there are several studies to identify the customer credit risk, qualifying them as good or bad payers, helping to make a decision to grant credit, there is few research studying the credit recovery, when the delinquency occurs. [2]

In [3], the author conducted a study evaluating 41 publications on the award of credit since 2006, all of them using classifiers to categorize customers as good or bad payers. Those works were organized into three categories of classifiers: individuals; homogeneous ensemble; and heterogeneous ensemble classifiers. Most of the algorithms used were implemented through logistic regression and decision trees, with their use of boosting, bagging and forest variants.

The Table 2 lists the eight datasets that were used in [3] to verify the performance of each of the 41 models proposed, evaluating them from the standpoint of 6 indicators: Area Under the Receiver Operating Curve (AUC), percentage correctly classified (PCC), partial Gini index, H-measure, Brier Score (BS) and Kolmogorov-Smirnov (KS).

Table 2: Datasets used in [3].

\begin{tabular}{|l|r|r|r|}
\hline Name & Samples & Features & Debtors \% \\
\hline AC & 690 & 14 & 44.5 \\
\hline GC & 1000 & 20 & 30.0 \\
\hline Th02 & 1225 & 17 & 26.4 \\
\hline Bene 1 & 3123 & 27 & 66.7 \\
\hline Bene 2 & 7190 & 28 & 30.0 \\
\hline UK & 30000 & 14 & 4.0 \\
\hline PAK & 50000 & 37 & 26.1 \\
\hline GMC & 150000 & 12 & 6.7 \\
\hline
\end{tabular}

\footnotetext{
${ }^{1}$ Documentation available at http://docs.h2o.ai/h2o/latest-stable/index.html

${ }^{2} \mathrm{H} 2 \mathrm{O}$ is an open source machine learning platform, available at www.h2o.ai
} 
In [4], the author presents AUC as an indicator that represent how well classified were the data, independent of its distribution or misclassification costs. PCC is an overall accuracy measure that indicates the percentage of outcomes that were correctly classified.[5]

A score was assigned to each algorithm, referring to the classification received in the comparison between them within the same performance measure . For example, the algorithm K-means was in 12th place considering the AUC indicator, while the KNN was in 29th place. Thus, the scores attributed to them were 12 and 29, respectively. Then, the algorithms were ordered by the average of all metrics, where the 1 st place were the algorithm that obtained the lowest score.

The heterogeneous multi-classifiers presented a better performance, although the performance between the three categories was very similar.

The Table 3 presents the results of the benchmark, indicating that the HCES-Bag algorithm obtained the highest AUC result, while the AVG-W and Gasen algorithms reached $80.7 \%$ of the PCC.

Table 3: State of Art - Models Comparison - Adapted from [3]

$\begin{array}{cllll} & \text { Algorithm } & \text { AUC } & \text { PCC } \\ \text { Heterogeneous Ensemble } & \text { HCES-Bag } & 0.932 & 80.2 \\ & \text { AVG W } & 0.931 & 80.7 \\ & \text { GASEN } & 0.931 & 80.7 \\ \text { Homogeneous Ensemble } & \text { RF } & 0.931 & 78.9 \\ & \text { BagNN } & 0.927 & 80.2 \\ & \text { Boost } & 0.93 & 77.2 \\ \text { Individual } & \text { LR } & 0.931 & 70.84 \\ & \text { LDA } & 0.929 & 78.4 \\ & \text { SVM-Rbf } & 0.925 & 79.9\end{array}$

\section{Methodology and Infrastructure Setup}

This section presents the methodology used in this study, which was segmented in stages according to the phases proposed by CRISP-DM [6]. The result of each phase is described in the next Section.

Training model environment - The models were trained on the H2O.ai platform, in a cluster formed by 5 virtual machines on the same subnet and with the same configuration. Their operating system was Red Hat Enterprise Linux 6.864 bits, with 34 cores and 80 GB of RAM. It were used H2O.ai version 3.10.4.5 and $\mathrm{R}$ version 3.3.0. It were allocated $44 \mathrm{~GB}$ of RAM and all cores of each machine, reaching a total of 170 cores and $220 \mathrm{~GB}$ of RAM.

The training dataset consisted of about 40 million copies, requiring a robust platform to be made available for the processing of this data.

The Figure 2 shows the CPU meter of the H2O.ai cluster in action at the moment of the training models. It shows the percentage of use of the processors of each machine, identified by the final number of its IP address (174 to 178 ) and the port number where the service was running (54321). The intensive use of the 170 available cores shown in the Figure 2 reinforces the need for a robust platform.

Each vertical bar represents 1 core and the colors represent the type of process executed: idle time (blue), user time (green) and system time (red).

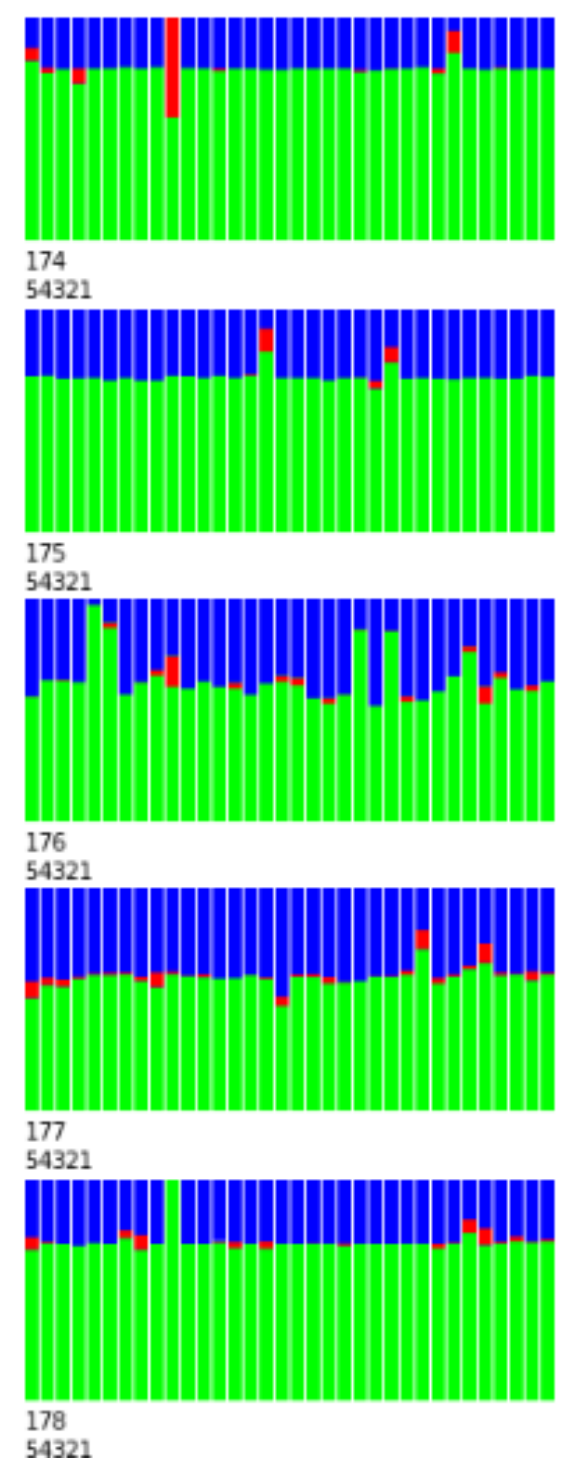

Figure 2: Cluster $\mathrm{H} 2 \mathrm{O}$ in action

\section{Results}

In this sections, the results of the CRISP-DM phases are detailed: Data Understanding, Data preparation, Modeling, Evaluation and Implementation.

\subsection{Data Understanding}

The dataset was obtained by the extraction of information from legacy systems and customers relationship data marts. It has information about customers accounting,demographic and financial data. The dataset had 28 features and 1 label that indicates the recovery of the respective credit operation. The 
Tables 4 and 5 present these 28 characteristics organized by categorical and numerical features.

\begin{tabular}{|l|l|}
\multicolumn{2}{l|}{ Table 4: Numeric features } \\
\hline Features & \multicolumn{1}{c|}{ Description } \\
\hline V1 & $\begin{array}{l}\text { Number of days of delinquency. } \\
\text { end of the contract. }\end{array}$ \\
\hline V3 & Contract value. \\
\hline V4 & Amount of the outstanding balance. \\
\hline V5 & $\begin{array}{l}\text { Amount PDA provisioned for the } \\
\text { contract. }\end{array}$ \\
\hline V6 & $\begin{array}{l}\text { Percentage loss expected for the con- } \\
\text { tract. }\end{array}$ \\
\hline V7 & $\begin{array}{l}\text { Quantity of products owned by the } \\
\text { customer. }\end{array}$ \\
\hline V8 & $\begin{array}{l}\text { Time of customer relationship with } \\
\text { the Bank. }\end{array}$ \\
\hline V9 & Customer age. \\
\hline V10 & Customer income. \\
\hline V11 & $\begin{array}{l}\text { Customer total contribution margin } \\
\text { amount. }\end{array}$ \\
\hline V12 & $\begin{array}{l}\text { Value of Gross Domestic Product per } \\
\text { capita }\end{array}$ \\
\hline
\end{tabular}

Table 5: Categorical features

\begin{tabular}{|l|l|}
\hline Features & \multicolumn{1}{|c|}{ Description } \\
\hline VC1 & Customer portfolio type. \\
\hline VC2 & Customer behavioral segment. \\
\hline VC3 & Product. \\
\hline VC4 & Product modality. \\
\hline VC5 & Structured operation indicator. \\
\hline VC6 & $\begin{array}{l}\text { Management level that approved the } \\
\text { operation. }\end{array}$ \\
\hline VC7 & Transaction risk credit. \\
\hline VC8 & Range of past delays. \\
\hline VC9 & PDA lock indicator. \\
\hline VC10 & $\begin{array}{l}\text { Customer relationship with the } \\
\text { bank. }\end{array}$ \\
\hline VC11 & Client instruction level. \\
\hline VC12 & Customer gender. \\
\hline VC13 & Nature of customer occupation. \\
\hline VC14 & Customer registration status. \\
\hline VC15 & Customer's age group. \\
\hline VC16 & Age group of relationship time. \\
\hline
\end{tabular}

For the data understanding, the analysis began in July 2016 containing all credit operations contracts, regardless of the contracted product, with more than 14 days in arrears. In addition, transactions with the highest risk were considered as already lost contracts by our business specialists and removed from our dataset.

For definition of the label, the delay reduction indicator, the following operation was performed, considering that the data of the delayed operations were used in July 2016:
- Delay Reduction Indicator $=1$, for all transactions that showed a reduction in the number of days overdue in the subsequent month, that is, in August 2016, or that their debit balances have been reduced.

- Delay Reduction Indicator $=0$, otherwise, that is, presenting a delay or debit balance in August 2016 equivalent to or greater than that observed in July 2016.

The Table 6 presents the summary of transactions in the month of July 2016, which resulted in a base with $4,514,029$ contracts. Of this total, only 271,193 $(6.01 \%)$ were recovered.

Table 6: Dataset July 2016

\begin{tabular}{|r|r|}
\hline Not recovered & Recovered \\
\hline $4,242,836$ & 271,193 \\
\hline $93.99 \%$ & $6.01 \%$ \\
\hline Samples & $4,514,029$ \\
\hline
\end{tabular}

The bank has several strategies for credit recovery, according to the customer profile and the category of the credit operation, grouping them with distinct trading rules. Existing segments are divided into massive and individual strategies. Massive strategies are implemented for segments that have a known behavior pattern, whereas individual strategies cover operations that have atypical or special characteristics which require a case-by-case analysis to perform a collection and recovery.

Based on this information, the dataset was splitted into segments compatible with the institution's recovery strategies, grouping similar products and customer segments with characteristics in common removing from the study the segments that have an individualized trading strategy. The Table 7 lists the 11 segments that will be worked on in this study, in addition to the Individualized Strategy segment, which was removed from the study.

\subsection{Data Preparation}

In this study, the analysis were performed only in the first 3 segments, Mortgage Loan I, II and III. The remaining segments are in the final analysis phase and will be presented at a later time.

Then, the data preparation was started, analyzing each one of the segments, preparing the data sets for the modeling phase.

The Tables 8,10 and 12 present the summary of descriptive analysis of the numerical features of segments Mortgage Loan I, II and III, respectively. In these tables the data of quartiles and Kendall's Tau [7] of each feature are presented.

The Tables 9, 11 and 13 present the summary of the descriptive analysis of the categorical variables, listing the Kendall's Tau and the number of levels of each feature. 
Table 7: Credit Operations Segments

\begin{tabular}{|l|r|r|r|r|r|}
\hline \multirow{2}{*}{\multicolumn{1}{|c|}{ Segment }} & \multicolumn{4}{|c|}{$\begin{array}{c}\text { Credit } \\
\text { Recovered }\end{array}$} & \multirow{2}{*}{ Samples } \\
\cline { 2 - 5 } & \multicolumn{1}{|c|}{ No } & \multicolumn{1}{c|}{ Yes } & \\
\cline { 2 - 5 } & \multicolumn{1}{|c|}{ Qty } & \multicolumn{1}{c|}{ Qty } & \multicolumn{1}{c|}{$\%$} \\
\hline Mortgage Loan I & 41,398 & 70.45 & 17,365 & 29.55 & 58,763 \\
\hline Mortgage Loan II & 400 & 73.94 & 141 & 26.06 & 541 \\
\hline Mortgage Loan III & 3,537 & 78.11 & 991 & 21.89 & 4,528 \\
\hline Vehicle Financing I & 12,115 & 87.90 & 1,667 & 12.10 & 13,782 \\
\hline Vehicle Financing II & 32,357 & 86.63 & 4,993 & 13.37 & 37,350 \\
\hline Agribusiness & 258,618 & 98.84 & 3,021 & 1.16 & 261,639 \\
\hline Social Business & 137,474 & 93.53 & 9,504 & 6.47 & 146,978 \\
\hline Credit Card I & 17,124 & 98.92 & 187 & 1.08 & 17,311 \\
\hline Credit Card II & 454,864 & 98.56 & 6,661 & 1.44 & 461,525 \\
\hline Other Operations Income I & 186,572 & 96.53 & 6,714 & 3.47 & 193,286 \\
\hline Other Operations Income II & $2,668,890$ & 92.96 & 201,977 & 7.04 & $2,870,867$ \\
\hline Individualized Strategy & 429,487 & 95.98 & 17,972 & 4.02 & 447,459 \\
\hline
\end{tabular}

Table 8: Mortgage Loan I - Numerical Features

\begin{tabular}{|l|r|r|r|r|r|r|r|}
\hline Feature & \multicolumn{1}{|c|}{ Min } & 1QT & Median & \multicolumn{1}{c|}{ Avg } & \multicolumn{1}{c|}{ 3QT } & \multicolumn{1}{c|}{ Max } & Kendall's Tau \\
\hline V1 & 15 & 20 & 51 & 87.43 & 112 & 624 & -0.29 \\
\hline V2 & 0 & 10,180 & 10,420 & 10,290 & 10,670 & 11,620 & -0.03 \\
\hline V3 & 0 & 0.1 & 0.13 & 0.17 & 0.27 & 0.67 & -0.02 \\
\hline V4 & 0 & 1 & 2 & 3.94 & 5 & 26 & 0.06 \\
\hline V5 & 17 & 25 & 29 & 31.28 & 36 & 73 & 0.03 \\
\hline V6 & 1 & 3 & 4 & 4.45 & 5 & 37 & 0.08 \\
\hline V7 & 14,790 & 74,400 & 87,460 & 86,270 & 97,470 & 164,800 & 0.01 \\
\hline V8 & $-124,400$ & -390.8 & 156.7 & $-1,397$ & 256.3 & 183,400 & 0.38 \\
\hline V9 & 0 & 1,349 & 3,221 & 3,712 & 5,525 & 46,040 & 0.04 \\
\hline V10 & 0 & 888.1 & 2,670 & 19,090 & 20,960 & 173,700 & -0.17 \\
\hline V11 & 0 & 1,586 & 1,700 & 1,877 & 2,000 & 20,000 & 0.03 \\
\hline V12 & 0.68 & 74,920 & 88,720 & 87,250 & 99,510 & 173,500 & 0.00 \\
\hline
\end{tabular}

\subsection{Modeling}

For each dataset, 4 predictive models were elaborated, using the $\mathrm{H} 2 \mathrm{O}$ platform integrated to the $\mathrm{R}$, using the algorithms Generalized Linear Models (GLM), Gradient Boosting Method (GBM), Random Forest (DRF) and Deep Learning (DL). The first three algorithms were chosen because they represent the techniques most used in the calculation of credit risk, which performs a classification task very similar in [8]. The algorithm DL was used to verify its behavior in a knowledge area not yet explored, but with expectation of good suitability due to the use of a great amount of variables. 9]

The datasets of the Mortgage Loan I and III segments were splitted into 3 parts: $70 \%$ for training, $20 \%$ for validation and $10 \%$ for testing. Due to the small number of observations in the Mortgage Loan II, this dataset was splitted only in training and validation in a proportion of $80 \%$ and $20 \%$, respectively. The next subsections present the evaluation results for each segment.

\subsubsection{Mortgage Loan I}

- GLM - This algorithm obtained an AUC = 0.7774755 and a PCC of $66.53 \%$, as shown in the Figure 3 and in the Table 14

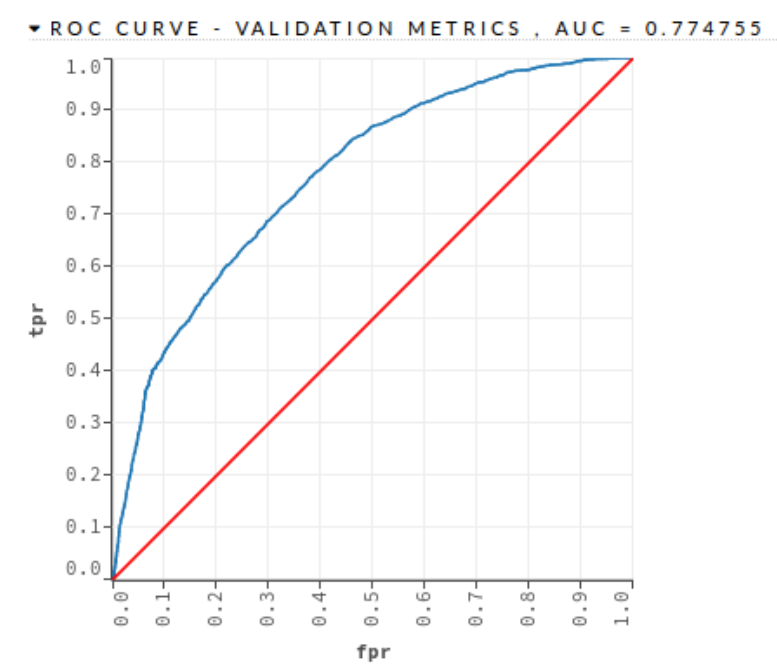

Figure 3: Mortgage Loan I - GLM - Validation Dataset AUC 
Table 9: Mortgage Loan I - Categorical Features

\begin{tabular}{|l|r|r|}
\hline Feature & Kendall's Tau & Number of levels \\
\hline VC1 & 0.23 & 6 \\
\hline VC2 & 0.02 & 5 \\
\hline VC3 & -0.09 & 3 \\
\hline VC4 & -0.21 & 9 \\
\hline VC5 & 0.03 & 12 \\
\hline VC6 & 0.06 & 7 \\
\hline VC7 & -0.01 & 2 \\
\hline VC8 & 0.03 & 5 \\
\hline VC9 & -0.04 & 16 \\
\hline VC10 & 0.00 & 4 \\
\hline VC11 & 0.05 & 8 \\
\hline VC13 & -0.21 & 9 \\
\hline VC14 & 0.01 & 4 \\
\hline VC15 & -0.02 & 18 \\
\hline VC16 & 0.00 & 2 \\
\hline
\end{tabular}

Table 10: Mortgage Loan II - Numerical Features

\begin{tabular}{|l|r|r|r|r|r|r|r|}
\hline Feature & \multicolumn{1}{|c|}{ Min } & \multicolumn{1}{|c|}{ QT } & Median & \multicolumn{1}{c|}{ Avg } & \multicolumn{1}{c|}{ Q T } & Max & Kendall's Tau \\
\hline V1 & 15 & 21 & 48 & 89 & 113 & 507 & -0.15 \\
\hline V2 & 0 & 1,626 & 2,928 & 3,037 & 4,076 & 6,776 & -0.14 \\
\hline V3 & 0 & 0 & 0 & 0 & 0 & 1 & 0.09 \\
\hline V4 & 2 & 6 & 6 & 10 & 13 & 31 & 0.03 \\
\hline V5 & 30 & 42 & 48 & 49 & 55 & 85 & 0.02 \\
\hline V6 & 1 & 4 & 6 & 7 & 9 & 36 & -0.04 \\
\hline V7 & 4,400 & 28,000 & 45,000 & 59,560 & 70,560 & 240,000 & -0.05 \\
\hline V8 & $-31,770$ & -148 & 91 & -650 & 371 & 25,070 & 0.30 \\
\hline V9 & 0 & 4,990 & 6,190 & 6,663 & 9,099 & 15,260 & 0.08 \\
\hline V10 & 0 & 173 & 650 & 8,744 & 10,230 & 116,000 & -0.20 \\
\hline V11 & 0 & 1,598 & 2,965 & 5,082 & 5,553 & 128,900 & -0.11 \\
\hline V12 & 0 & 9,316 & 20,500 & 36,670 & 47,120 & 215,200 & -0.14 \\
\hline
\end{tabular}

- DRF - This algorithm was implemented with 500 trees and a maximum depth of 7 . The DRF algorithm obtained an AUC $=0.880589$ and a PCC $=75.85 \%$, as shown in the Figure 4 and in the Table 14

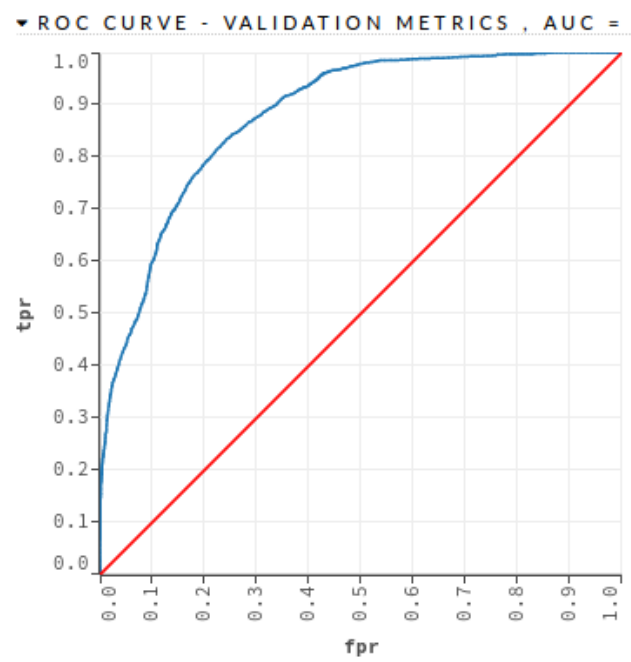

Figure 4: Mortgage Loan I - DRF - Validation Dataset AUC
- DL - Deep Learning This algorithm was implemented with 2 hidden layers with 200 neurons each one. The DRF algorithm obtained an AUC $=0.898203$ and a PCC $=79.22 \%$, as shown in the Figure 5 and in the Table 14.

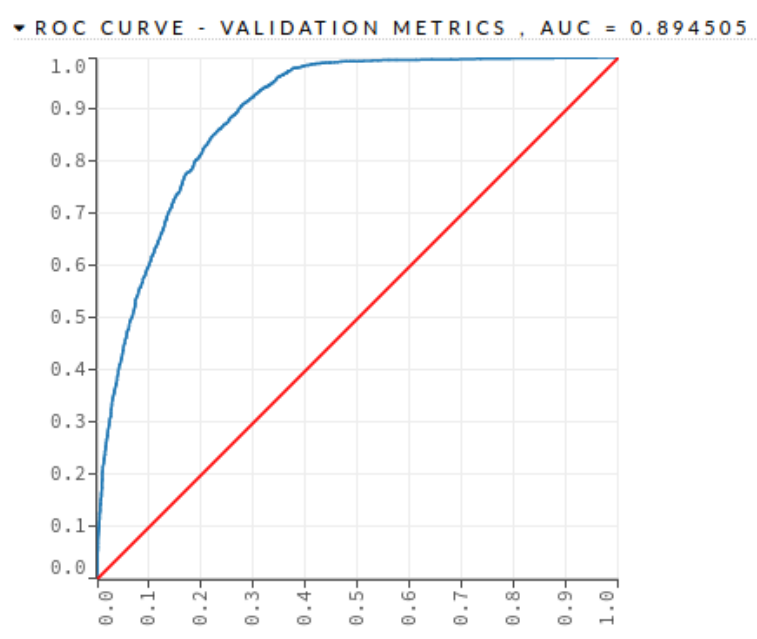

Figure 5: Mortgage Loan I - DL - Validation Dataset AUC 
Table 11: Mortgage Loan II - Categorical Features

\begin{tabular}{|l|r|r|}
\hline Features & Kendall's Tau & Number of levels \\
\hline VC1 & 0.11 & 4 \\
\hline VC2 & 0.13 & 3 \\
\hline VC4 & -0.19 & 8 \\
\hline VC5 & 0.01 & 10 \\
\hline VC6 & 0.03 & 6 \\
\hline VC7 & -0.04 & 2 \\
\hline VC8 & 0.10 & 5 \\
\hline VC9 & 0.06 & 10 \\
\hline VC11 & -0.15 & 5 \\
\hline VC13 & -0.19 & 8 \\
\hline VC14 & 0.10 & 4 \\
\hline VC15 & -0.06 & 13 \\
\hline
\end{tabular}

Table 12: Mortgage Loan III - Numerical Features

\begin{tabular}{|l|r|r|r|r|r|r|r|}
\hline Feature & \multicolumn{1}{|c|}{ Min } & \multicolumn{1}{c|}{ 1QT } & Median & \multicolumn{1}{c|}{ Avg } & \multicolumn{1}{c|}{ 3QT } & \multicolumn{1}{c|}{ Max } & Kendall's Tau \\
\hline V1 & 15 & 30 & 81 & 131 & 181 & 511 & -0.31 \\
\hline V2 & 0 & 4,876 & 7,530 & 6,616 & 8,203 & 10,910 & -0.01 \\
\hline V3 & 0.00 & 0.02 & 0.05 & 0.06 & 0.07 & 0 & 0.00 \\
\hline V4 & 0 & 5 & 9 & 11 & 15 & 54 & -0.02 \\
\hline V5 & 20 & 35 & 43 & 44 & 52 & 78 & -0.06 \\
\hline V6 & 2 & 6 & 8 & 10 & 11 & 71 & 0.05 \\
\hline V7 & 20,000 & 100,000 & 142,500 & 188,700 & 213,800 & $3,000,000$ & -0.07 \\
\hline V8 & $-257,600.00$ & $-5,476.00$ & -930.00 & $-9,087.00$ & 257.70 & 199,600 & 0.36 \\
\hline V9 & 0 & 2,769 & 4,660 & 4,968 & 6,485 & 46,040 & 0.01 \\
\hline V10 & 0 & 3,317 & 14,200 & 51,540 & 53,470 & $1,212,000$ & -0.20 \\
\hline V11 & 0 & 2,280 & 5,542 & 10,700 & 11,130 & 337,600 & -0.01 \\
\hline V12 & 250 & 88,900 & 134,500 & 177,500 & 203,200 & $3,084,000$ & -0.08 \\
\hline
\end{tabular}

- GBM - This algorithm was implemented with 500 trees and a maximum depth of 7. The GBM algorithm obtained an AUC $=0.988574$ and a PCC $=93.90 \%$, as shown in the Figure 6 and in the Table 14

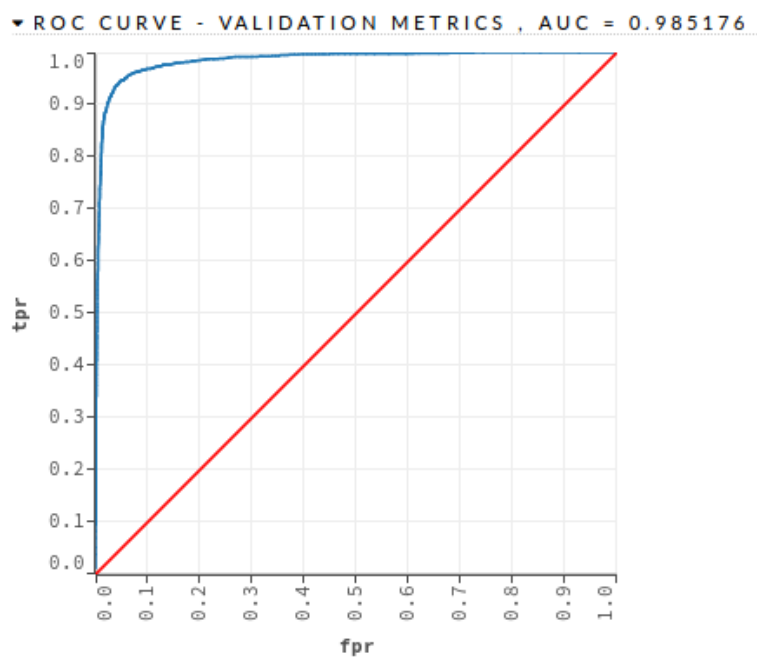

Figure 6: Mortgage Loan I - GBM - Validation Base AUC

\subsubsection{Mortgage Loan II}

Because of the small number of records, this dataset was splitted only in training and testing, in the ratio of 80:20, and validation was performed through cross validation with 10 folds.

- GLM - This algorithm obtained an AUC = 0.848474 and $\mathrm{PCC}=65.51 \%$, as shown in the Figure 7 and in the Table 15

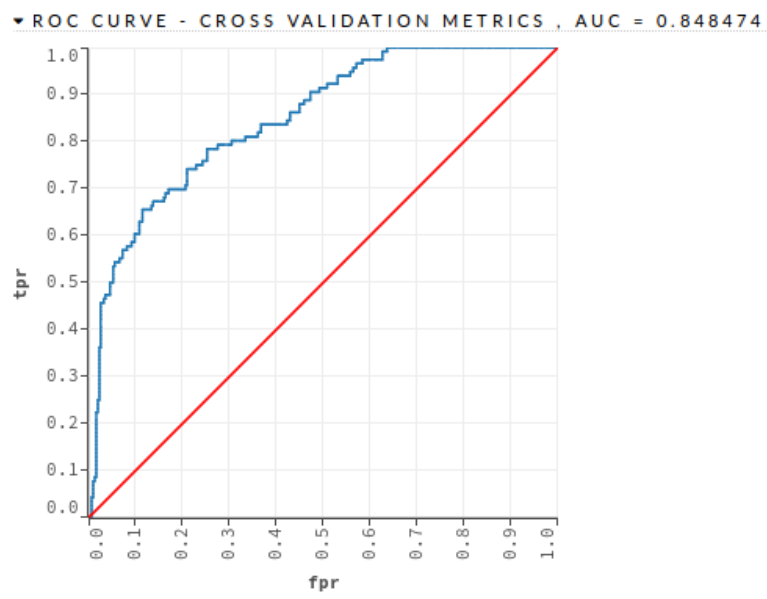

Figure 7: Mortgage Loan II - GLM - Validation Dataset AUC 
Table 13: Mortgage Loan III - Categorical Features

\begin{tabular}{|l|r|r|}
\hline Feature & Kendall's Tau & Number of levels \\
\hline VC1 & 0.16 & 6 \\
\hline VC2 & 0.00 & 4 \\
\hline VC3 & -0.24 & 2 \\
\hline VC4 & -0.21 & 33 \\
\hline VC5 & -0.06 & 12 \\
\hline VC6 & -0.02 & 7 \\
\hline VC7 & -0.03 & 2 \\
\hline VC8 & 0.00 & 5 \\
\hline VC9 & -0.02 & 16 \\
\hline VC10 & -0.10 & 5 \\
\hline VC11 & 0.03 & 7 \\
\hline VC12 & 0.00 & 2 \\
\hline VC13 & -0.21 & 9 \\
\hline VC14 & 0.00 & 5 \\
\hline VC15 & -0.04 & 17 \\
\hline VC16 & 0.17 & 2 \\
\hline
\end{tabular}

Table 14: Mortgage Loan I - Confusion Matrix

\begin{tabular}{|l|r|r|r|r|r|}
\hline Algorithm & & \multicolumn{1}{c|}{$\mathbf{0}$} & $\mathbf{1}$ & Err \% & PCC \\
\hline \multirow{3}{*}{ GLM } & 0 & 5003 & 3050 & 37.87 & \\
\cline { 2 - 6 } & 1 & 776 & 2603 & 22.96 & \\
\cline { 2 - 6 } & Total & 5779 & 5653 & 33.46 & 66.52 \\
\hline \multirow{3}{*}{ DRF } & 0 & 6638 & 1415 & 17.57 & \\
\cline { 2 - 6 } & 1 & 816 & 2563 & 24.14 & \\
\cline { 2 - 6 } & Total & 7454 & 3978 & 19.51 & 75.85 \\
\hline \multirow{3}{*}{ GBM } & 0 & 6290 & 1763 & 21.89 & \\
\cline { 2 - 6 } & 1 & 517 & 2862 & 15.39 & \\
\cline { 2 - 6 } & Total & 6897 & 4625 & 19.94 & 79.22 \\
\cline { 2 - 6 } & $\mathbf{0}$ & $\mathbf{7 7 7 0}$ & $\mathbf{2 8 3}$ & $\mathbf{3 . 5 1}$ & \\
\cline { 2 - 6 } & Total & $\mathbf{8 0 0 8}$ & $\mathbf{3 4 2 4}$ & $\mathbf{3 . 5 5}$ & $\mathbf{9 3 . 9 0}$ \\
\hline
\end{tabular}

Table 15: Mortgage II - Confusion Matrix

\begin{tabular}{|l|c|r|r|r|r|}
\hline Algorithm & & $\mathbf{0}$ & \multicolumn{1}{c|}{$\mathbf{1}$} & Err \% & PCC \\
\hline \multirow{3}{*}{ GLM } & 0 & 270 & 35 & 11.47 & \\
\cline { 2 - 6 } & 1 & 40 & 76 & 34.48 & \\
\cline { 2 - 6 } & Total & 310 & 111 & 17.81 & 65.51 \\
\hline \multirow{3}{*}{ DRF } & 0 & 302 & 3 & 0.98 & \\
\cline { 2 - 6 } & 1 & 17 & 99 & 14.65 & \\
\cline { 2 - 6 } & Total & 319 & 102 & 4.75 & 85.34 \\
\hline \multirow{3}{*}{ DL } & $\mathbf{0}$ & $\mathbf{2 9 7}$ & $\mathbf{8}$ & 2.62 & \\
\cline { 2 - 6 } & $\mathbf{1}$ & $\mathbf{1 0}$ & $\mathbf{1 0 6}$ & $\mathbf{8 . 6 2}$ & \\
\cline { 2 - 6 } & Total & $\mathbf{3 0 7}$ & $\mathbf{1 1 4}$ & 4.27 & $\mathbf{9 1 . 3 7}$ \\
\hline \multirow{3}{*}{ GBM } & 0 & 301 & 4 & 1.31 & \\
\cline { 2 - 6 } & 1 & 16 & 100 & 13.79 & \\
\cline { 2 - 6 } & Total & 317 & 104 & 4.75 & 86.20 \\
\hline
\end{tabular}

- DRF - This algorithm was implemented with 500 trees and a maximum depth of 7 . The DRF algorithm obtained an AUC $=0.977982$ and a PCC $=93.10 \%$, as shown in the Figure 8 and in the Table 15

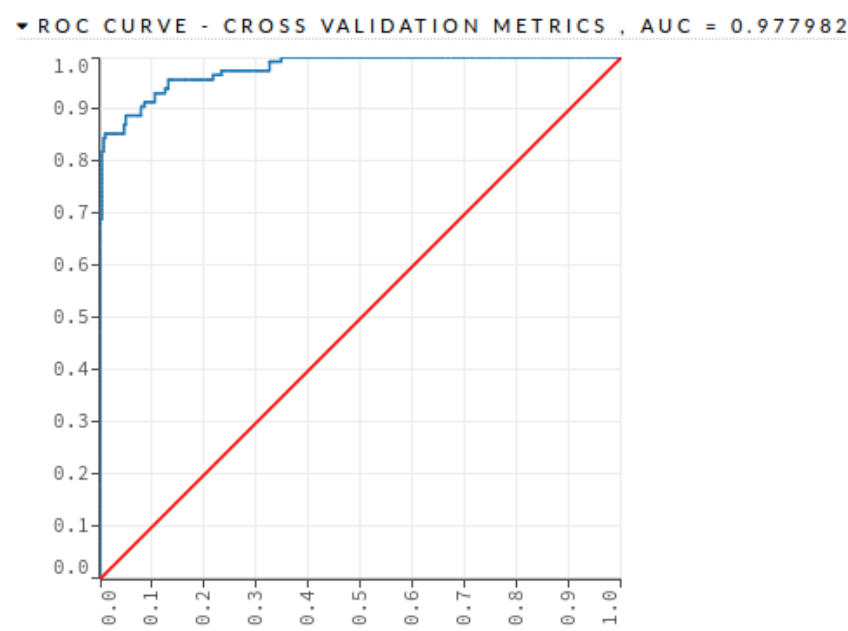

Figure 8: Mortgage Loan II - DRF - Validation Dataset AUC

- DL - This algorithm was implemented with 2 hidden layers with 200 neurons each one. The DRF algorithm obtained an AUC $=0.956868$ 
and a PCC $=91.37 \%$, as shown in the Figure 5 and in the Table 15

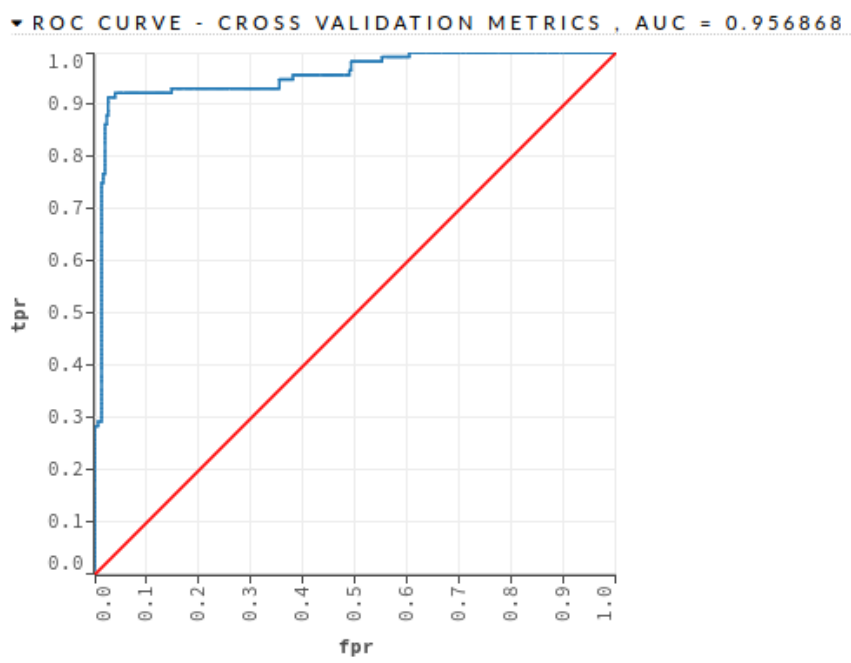

Figure 9: Mortgage Loan II - DL - Validation Dataset AUC

- GBM - This algorithm was implemented with 500 trees and a maximum depth of 7 . The GBM algorithm obtained an AUC $=0.972640$ and a PCC $=86.20 \%$, as shown in the Figure 10 and in the Table 15

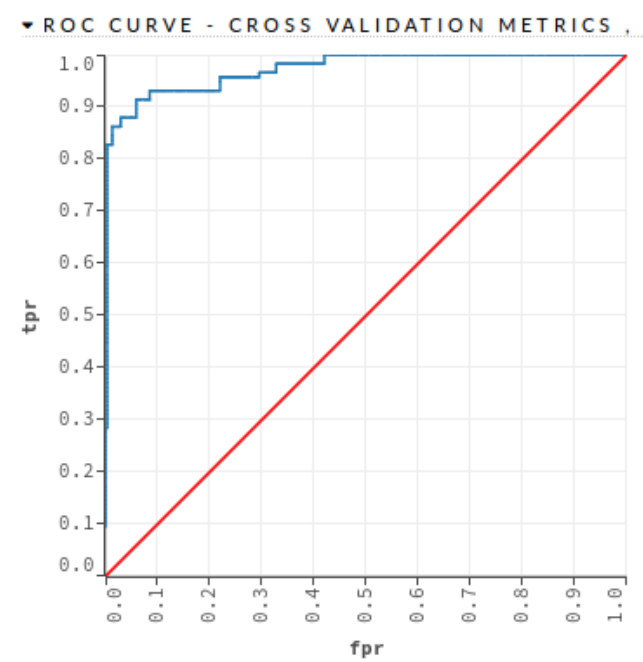

Figure 10: Mortgage Loan II - GBM - Validation Dataset AUC

\subsubsection{Mortgage Loan III}

- DRF - This algorithm was implemented with 500 trees and a maximum depth of 7 . The DRF algorithm obtained an AUC $=0.950718$ and a PCC $=83.51 \%$, as shown in the Figure 11 and in the Table 16

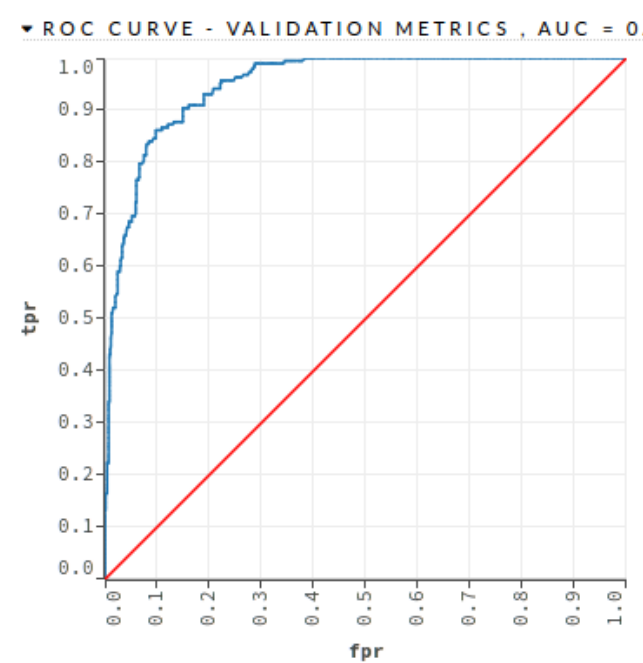

Figure 11: Mortgage Loan III - DRF - Validation Dataset AUC

- DL - This algorithm was implemented with 2 hidden layers with 200 neurons each one. The DRF algorithm obtained an AUC $=0.939082$ and a PCC $=78.20 \%$, as shown in the Figure 12 and in the Table 16

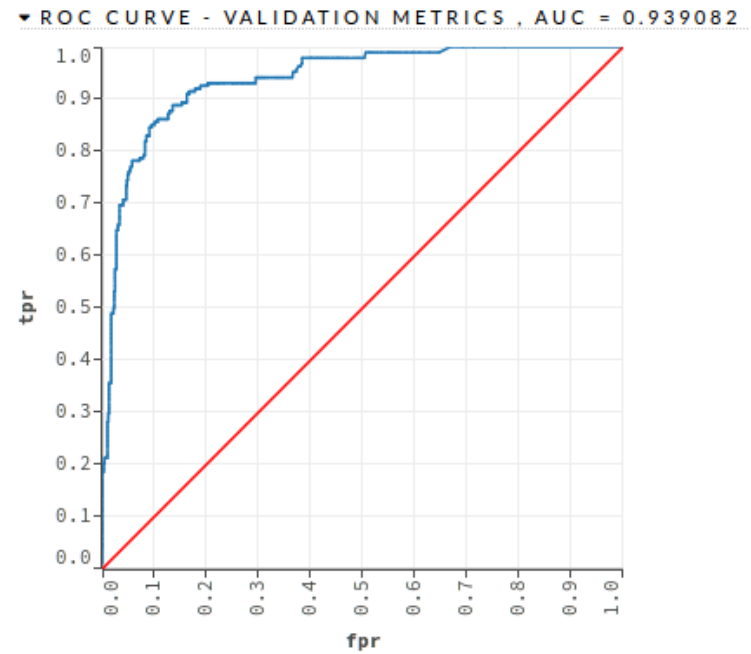

Figure 12: Mortgage Loan III - DL - Validation Dataset AUC

- GBM - This algorithm was implemented with 500 trees and a maximum depth of 7. The GBM algorithm obtained an AUC $=0.955728$ and a PCC $=98.93 \%$, as shown in the Figure 13 and in the Table 16 


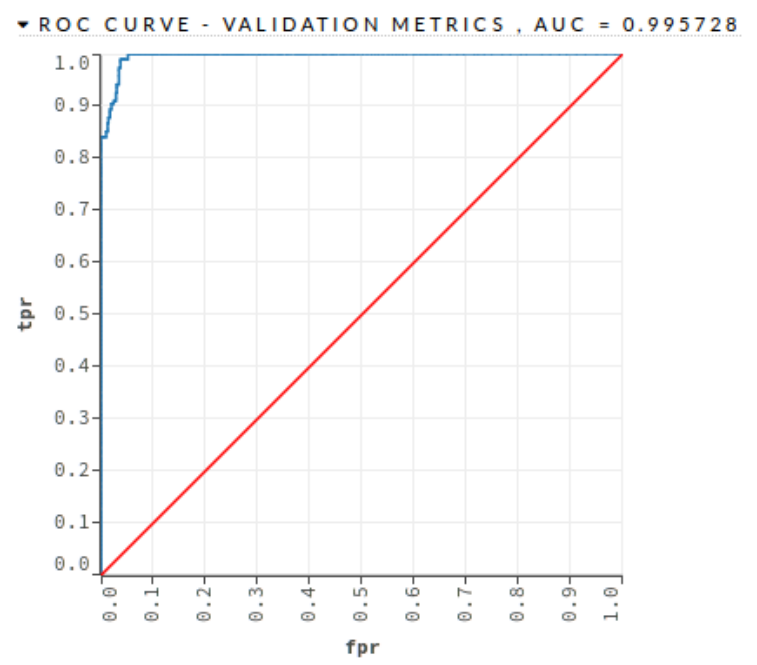

Figure 13: Mortgage Loan III - GBM - Validation Dataset AUC

- GLM - This algorithm obtained an AUC = 0.814560 and $\mathrm{PCC}=60.10 \%$, as shown in the Figure 14 and in the Table 16

Table 16: Mortgage Loan III - Confusion Matrix

\begin{tabular}{|l|r|r|r|r|}
\hline Algorithm & \multicolumn{1}{|c|}{$\mathbf{0}$} & \multicolumn{1}{|c|}{$\mathbf{1}$} & Err \% & PCC \\
\hline \multirow{4}{*}{ GLM } & 601 & 95 & 13.64 & \\
\cline { 2 - 5 } & 75 & 113 & 39.89 & \\
\cline { 2 - 5 } & 676 & 208 & 19.23 & 60.10 \\
\hline \multirow{4}{*}{ DRF } & 640 & 56 & 8.04 & \\
\cline { 2 - 5 } & 31 & 157 & 16.48 & \\
\cline { 2 - 5 } & 671 & 213 & 9.84 & 83.51 \\
\hline \multirow{4}{*}{ DL } & 656 & 40 & 5.74 & \\
\cline { 2 - 5 } & 41 & 147 & 21.80 & \\
\cline { 2 - 5 } & 697 & 187 & 9.16 & 78.20 \\
\hline \multirow{3}{*}{ GBM } & $\mathbf{6 7 0}$ & $\mathbf{2 6}$ & $\mathbf{3 . 7 3}$ & \\
\cline { 2 - 5 } & 2 & $\mathbf{1 8 6}$ & $\mathbf{1 . 0 6}$ & \\
\cline { 2 - 5 } & $\mathbf{6 7 2}$ & $\mathbf{2 1 2}$ & $\mathbf{3 . 1 6}$ & $\mathbf{9 8 . 9 3}$ \\
\hline
\end{tabular}

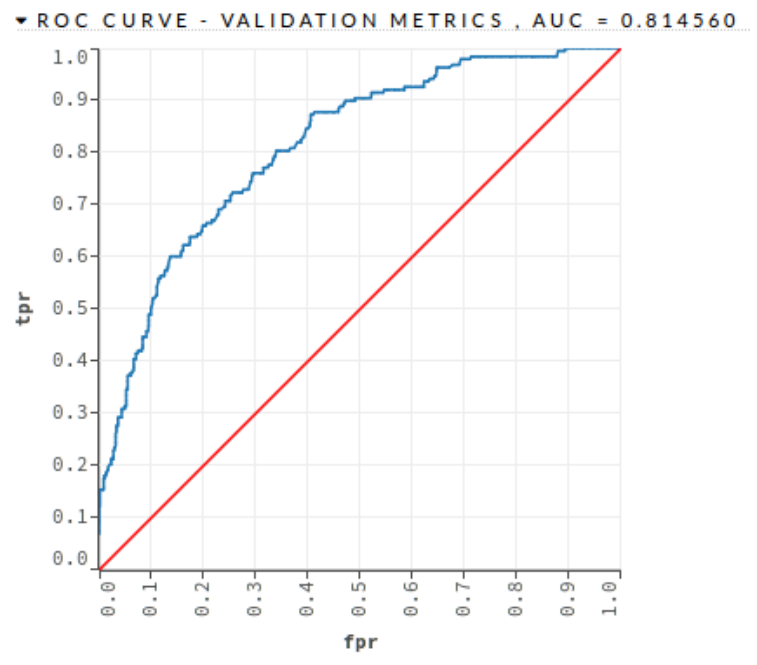

Figure 14: Mortgage Loan III - GLM - Validation Dataset AUC

\section{Conclusion}

The main objective of this study was to apply machine learning techniques to predict the probability of recovery of credit transactions, providing a list of defaulting clients with greater potential for regularization of their operations.

Studies were carried out on 3 segments of credit operations, which have different recovery strategies, the Mortgage Loan segments I, II and III. With the machine learning, it was possible to elaborate predictive models with great contribution to assist the Managers in the approach to their clients with operations in arrears.

Mortgage Loan I - The model with the highest recall was obtained with the GBM algorithm. In a total of 11,342 contracts in default, there were 3,424 contracts recovered. The model was able to correctly predict 3,141 contracts, reaching a recall of $92.86 \%$. Using the prioritization list generated by the model, the work of Bank Managers would be more assertive. In addition, the model correctly predicted $7,770(97.02 \%)$ contracts, out of 8,008 contracts that would not be recovered.

Mortgage Loan II - The model with the highest recall was obtained with the DL algorithm. In a total of 421 delinquent contracts, there were 116 contracts recovered. The model was able to correctly predict 106 contracts, reaching a recall of $94.38 \%$. In addition, the model correctly predicted 297 (96.74\%) contracts out of 307 contracts that would not be recovered.

Mortgage Loan III - The model with the highest recall was obtained with the GBM algorithm. In a total of 884 delinquent contracts, there were 212 contracts recovered. The model was able to accurately predict 186 contracts, reaching a recall of $98.94 \%$. In addition, the model correctly predicted 670 (99.70\%) contracts out of 672 contracts that would not be recovered.

The predictive models obtained from the analysis of the first three segments, out of a total of 11, have already shown a potential great benefit to the bank, effectively assisting its customers with delayed operations and avoiding unnecessary efforts in attempts in attempts of negotiation in contracts with low probability of recovering.

\subsection{Future Works}

The results obtained so far strengthen initiatives for the development of predictive models using machine learning techniques in the Bank studied.

With the increase in the efficiency of credit recovery, the Bank will benefit from the reduction in Allowance for Loan Losses (PDA), directly promoting positive results, with the reversal of provisions already made.

Thus, the study will be expanded to the 8 segments that have not yet been modeled, increasing the use of models obtained through machine learning techniques in credit recovery. In addition, the models al- 
ready obtained can be improved with the use of ensemble models.

\section{References}

[1] Rogerio G. Lopes, Rommel N. Carvalho, Marcelo Ladeira, and Ricardo S. Carvalho. Predicting Recovery of Credit Operations on a Brazilian Bank. pages 780-784. IEEE, December 2016.

[2] Sung Ho Ha. Behavioral assessment of recoverable credit of retailer's customers. Inf. Sci., 180(19):3703-3717, October 2010.

[3] Stefan Lessmann, Bart Baesens, Hsin-Vonn Seow, and Lyn C. Thomas. Benchmarking state-of-the-art classification algorithms for credit scoring: An update of research. European Journal of Operational Research, 247(1):124-136, 2015.

[4] Wouter Verbeke, Karel Dejaeger, David Martens, Joon Hur, and Bart Baesens. New insights into churn prediction in the telecommunication sector: A profit driven data mining approach. European Journal of Operational Research, 218(1):211 229,2012
[5] Karel Dejaeger, Frank Goethals, Antonio Giangreco, Lapo Mola, and Bart Baesens. Gaining insight into student satisfaction using comprehensible data mining techniques. European Journal of Operational Research, 218(2):548 - 562, 2012.

[6] Pete Chapman, Julian Clinton, Randy Kerber, Thomas Khabaza, Thomas Reinartz, Colin Shearer, and Rudiger Wirth. Crisp-dm 1.0 step-by-step data mining guide. Technical report, The CRISP-DM consortium, August 2000.

[7] Stephan Arndt, Carolyn Turvey, and Nancy C Andreasen. Correlating and predicting psychiatric symptom ratings: Spearmans $r$ versus kendalls tau correlation. Journal of psychiatric research, 33(2):97-104, 1999.

[8] Bart Baesens, Tony Van Gestel, Stijn Viaene, Maria Stepanova, Johan Suykens, and Jan Vanthienen. Benchmarking state-ofthe-art classification algorithms for credit scoring. Journal of the operational research society, 54(6):627-635, 2003.

[9] Yann LeCun, Yoshua Bengio, and Geoffrey Hinton. Deep learning. Nature, 521(7553):436-444, 2015. 